\title{
A thermodynamic unification of jamming
}

\author{
KEVIN LU1' E. E. BRODSKY² AND H. P. KAVEHPOUR ${ }^{1 *}$ \\ ${ }^{1}$ Department of Mechanical and Aerospace Engineering, University of California, Los Angeles, California 90095, USA \\ ${ }^{2}$ Department of Earth and Planetary Sciences, University of California, Santa Cruz, California 95064, USA \\ *e-mail: pirouz@seas.ucla.edu
}

Published online: 6 April 2008; doi:10.1038/nphys934

Fragile materials ${ }^{1}$ ranging from sand to fire retardant to toothpaste are able to exhibit both solid and fluid-like properties across the jamming transition. Unlike ordinary fusion, systems of grains, foams and colloids jam and cease to flow under conditions that still remain unknown. Here, we quantify jamming using a thermodynamic approach by accounting for the structural ageing and the shear-induced compressibility ${ }^{2}$ of dry sand. Specifically, the jamming threshold is defined using a non-thermal temperature ${ }^{3}$ that measures the 'fluffiness' of a granular mixture. The thermodynamic model, cast in terms of pressure, temperature and free volume, also successfully predicts the entropic data of five molecular glasses. Notably, the predicted configurational entropy averts the Kauzmann paradox ${ }^{4}$-an unresolved crisis where the configurational entropy becomes negative-entirely. Without any free parameters, the proposed equation-of-state also governs the mechanism of shear banding and the associated features of shear softening ${ }^{5,6}$ and thickness invariance ${ }^{2,7}$.

Despite their mundane appearance, granular materials exhibit a wide range of intriguing phenomena ${ }^{8,9}$. Dry sand, for instance, can deform readily ${ }^{9}$ but can also jam abruptly, for example, as observed in the sudden stoppage of flow in an hourglass or a salt shaker. The abruptness of jamming refers to the narrow range of packing fractions ${ }^{10}(0.62-0.64)$ under which the material no longer deforms. Molecular systems also exhibit similar jamming phenomena. For example, liquids such as wood glue become extremely viscous and resistant to flow when cooled within a narrow range of temperature ${ }^{11}\left(2-3^{\circ} \mathrm{C}\right)$ below the freezing point. This jamming behaviour shared by both granular fluids and viscous liquids is astonishing ${ }^{8,12,13}$ and suggestive of a common underlying mechanism, but thus far, a definitive theoretical connection remains unknown.

Jamming was defined ${ }^{14,15}$ as a means to unify all fragile systems ${ }^{1}$ and has been qualitatively described using three independent variables: pressure, packing fraction and an effective temperature ${ }^{13}$. It is known, however, that granular packings are metastable: any perturbation in the magnitude or the direction of the applied stress will cause structural ageing ${ }^{1,10}$, during which particles rearrange through irreversible compaction. It is thus problematic to neglect ageing and assume, for example, that the temperature at which jamming occurs can be defined by pressure and packing fraction alone. Still, many studies of fragile systems neglect the implications of ageing, possibly because of the narrow range in the temperature and packing density of glassy and granular systems near structural arrest. Here, we present a new perspective on jamming that includes a connection to the glass transition of viscous liquids. The proposed equation-of-state (EOS) will introduce jamming as path-dependent states definable by the stationary observables pressure, packing density and shear rate.
Recent shear flow experiments ${ }^{2}$ deduced the EOS of dense granular flows. We observed that the external pressure, $P$, in terms of shear rate, $\dot{\gamma}$, and the free volume ${ }^{16}, \varepsilon$, has the form

$$
P(\varepsilon, \dot{\gamma})=\frac{1}{\kappa_{1}} \ln \left[\frac{\varepsilon_{0}}{\varepsilon} \frac{1}{1-C \exp \left(-\kappa_{2} \dot{\gamma}\right)}\right] .
$$

For dry sand, the constants $\kappa_{1} \approx 7 \times 10^{-4} \mathrm{~Pa}^{-1}$ and $\kappa_{2} \approx 2 \times 10^{-5} \mathrm{~s}$. These constants also match values found independently from experimental data using the cyclic rule $^{2}$. The free volume $\varepsilon \equiv\left(V-V_{\mathrm{RCP}}\right)$ is the flowing shear-band volume $V$ referenced to its dynamic random-close-packing volume, $V_{\mathrm{RCP}}$. It is normalized by a fit value of the minimum free volume $\varepsilon_{0}$. As shown in Fig. 1, equation (1) indicates that the isochoric flows are shear weakening at intermediate shear velocities. The experimental data, spanning five decades from 0.001 to $10 \mathrm{rad} \mathrm{s}^{-1}$, reveal that the pressure dips and reaches a minimum between the quasi-static and grain-inertial regimes ${ }^{17}$. As may be expected, the weakening mechanism also applies to isobaric flows. Indeed, isobaric shear compacting is the counterpart to isochoric shear weakening; the solid volume fraction peaks within intermediate shear velocities. These isochoric and isobaric flow regimes, however, are interdependent; together, they constitute the transitional regime of granular flow. (A flow sweet spot is observed near $\dot{\gamma} \approx 200 \mathrm{~s}^{-1}$.)

The shear-softening scenario presented above has been observed in driven metallic ${ }^{5}$ and colloidal ${ }^{6}$ glasses, substances that are disordered solids that lack the periodicity of crystals. Why do granular fluids flow like glassy liquids? To explain, we measured the volume compaction of a sand column (radius $\approx 10 \mathrm{~cm}$ ) sheared at constant velocity. Figure 2 shows that granular compaction occurs at a rate that decreases nonlinearly in time with a decaying relaxation constant, $\tau$. The result is fitted using the KohlrauschWilliams-Watts ${ }^{4,18}$ equation,

$$
\frac{h(t)-h(\infty)}{h(0)-h(\infty)}=\exp \left[-\left(\frac{t}{\tau(T)}\right)^{\beta}\right] .
$$

The equation models the normalized change in the sand column height, $h(t)$, as a function of time, $t$, and the Kolrausch exponent, $\beta$. As noted previously ${ }^{18}$, the relaxation constant $\tau$ is Arrhenius at high temperatures, that is, $\tau \sim \exp \left(H / k_{\mathrm{B}} T\right)$ where $k_{\mathrm{B}}$ is the Boltzmann constant, $T$ is the thermal temperature and $H$ is the single 'void-hopping' activation energy ${ }^{11}$.

From Fig. 2, inset, we observed that the relaxation is defined by a stretched exponential with a Kolrauch exponent that approaches $\beta \approx 0.6$ as $\tau \rightarrow \infty$. The value of $\beta<1$ corresponds physically to the multiple relaxation mechanisms $s^{4,11,16}$ of granular compaction. Furthermore, its inverse dependence on $\tau$ signifies an increase of the apparent activation energy as packing density increases ${ }^{18}$, 


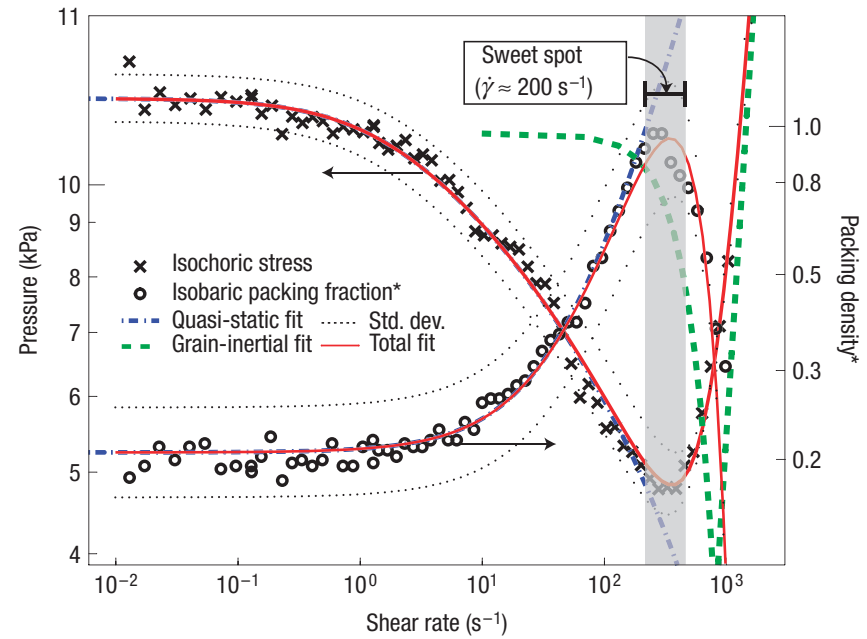

Figure $1 \mathrm{~A}$ log-log plot of granular rheology of isochoric pressure, $\boldsymbol{P}$, versus shear rate, $\dot{\gamma}$, and isobaric (normalized) packing-density ${ }^{*}, \varepsilon_{0} / \varepsilon$, versus $\dot{\gamma}$. Measurements are made using a torsional rheometer (AR-2000, TA Instruments). Compacted beach sand (grain size $\approx 438 \pm 188 \mu \mathrm{m}$, from Santa Monica) is confined concentrically while the top surface shears through logarithmically distributed velocities. The theoretical fit uses equation (1) with an extra grain-inertial term ${ }^{17}$, $M \rho D^{2} \dot{\gamma}^{2}$, in terms of grain density, $\rho$, averaged grain diameter, $D$, and fitting constant, $M$. The sweet spot signifies the optimum efficiency in achieving steady-state flow. Shear rate $\dot{\gamma}$ is calculated on the basis of a two-grain-diameter thickness ${ }^{2}$. The values for the isochoric fit are $C=0.990 \pm 0.004$,

$\kappa_{1}=7.3 \pm 0.4 \times 10^{-4} \mathrm{~Pa}^{-1}, \kappa_{2}=2.1 \pm 0.8 \times 10^{-5} \mathrm{~s}$ and $M=0.9 \pm 0.7 \times 10^{-3}$. For the isobaric fit, the values are $C=0.990 \pm 0.008, \kappa_{1}=7.0 \pm 0.3 \times 10^{-4} \mathrm{~Pa}^{-1}$, $\kappa_{2}=2.5 \pm 0.6 \times 10^{-5} \mathrm{~s}$ and $M=2.1 \pm 0.5 \times 10^{-3} ; \varepsilon_{0} \approx 4.1 \times 10^{-9} \mathrm{~m}^{3}$ from all fits.

progressively hindering the process of particle rearrangement. This age-dependent activation energy of granular fluids is a type of non-Arrhenius behaviour ${ }^{11,16}$ reminiscent of heterogeneous glassy liquids.

The steady-state rheology of Fig. 1 is ageing and path independent, on the basis of the reversible branch of packing fraction $(0.62-0.64)$ observed experimentally ${ }^{10}$. The irreversible branch has an expected broader density range (0.555-0.645) (ref. 19). Figure 2, however, suggests that any constitutive model such as equation (1) must account for the continued compaction of granular flow even on much longer timescales $\left(\gg 10^{5} \mathrm{~s}\right)$. This implies that the phenomenological equation (1) is a special case of a more fundamental theory. To find it, we incorporate an ageing temperature, $\Theta$, into equation (1). The temperature $\Theta \sim 10^{-7} \mathrm{~J}$ (or equivalently, $\sim 10^{15} \mathrm{~K}$ as $k_{\mathrm{B}} \sim 10^{-23} \mathrm{~J} \mathrm{~K}^{-1}$ ) was measured by Song et al..$^{20}$ for millimetre-sized acrylic beads sheared in gravity, which is significant considering the fact that these particles (size $\gg 1 \mu \mathrm{m}$ ) are not subjected to thermal fluctuations. This fictive notion of hot and cold will explain a thermodynamic theory that governs the dynamics of both reversible shear flow and irreversible compaction.

To build a meaningful generalization of equation (1), we will incorporate $\Theta$ into the Helmholtz free energy of flowing sand, $F_{\text {sand }}$. Using the thermodynamic relation ${ }^{21}$ of $P=-(\mathrm{d} F / \mathrm{d} \varepsilon), F_{\text {sand }}$ is derived from equation (1) as

$$
\frac{F_{\text {sand }}}{N \Theta}=\ln \left(\frac{\varepsilon}{\varepsilon_{0}}\right)-1+\ln \left[1-C \exp \left(-\frac{\zeta}{\Theta}\right)\right] \text {. }
$$

The variables have been recombined into new quantities that are defined as follows: $N \equiv \varepsilon / \nu, \zeta \equiv \kappa_{2} \Theta \dot{\gamma}$ and $\Theta \equiv v / \kappa_{1}$ where

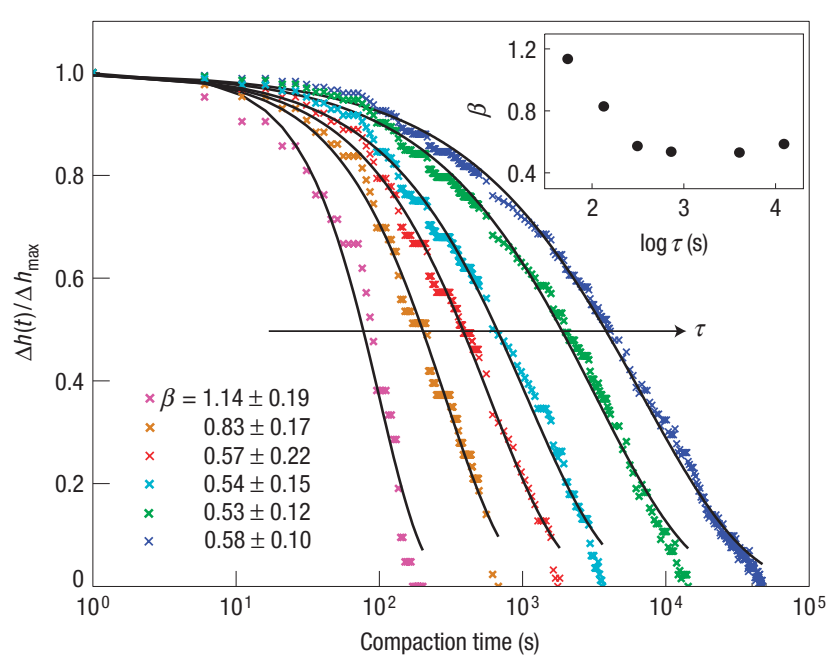

Figure 2 A semi-log plot of structural ageing of sheared granular mixture. Reduced column height of sand, $\left(h(t)-h\left(t_{\max }\right)\right) /\left(h(0)-h\left(t_{\max }\right)\right)$, versus compaction time, $t$; the fit uses the Kohlrausch-Williams-Watts relation of equation (2). The $\tau$-dependent relaxation, ageing, is non-Arrhenius; different activation energies correspond to different stages of structural ageing. The relaxation is also non-exponential; the inset shows that the Kohlrausch exponent, $\beta$, reaches a steady-state value of $\approx 0.5$ after $\approx 1 \mathrm{~h}$ to reflect the onset of cooperative particle motion representative of jammed systems. The non-Arrhenius and the non-exponential relaxations are reminiscent of the key features of glassy liquids. The sample uses $2.6 \mathrm{~g}$ of beach sand sheared at a constant velocity of $0.15 \mathrm{rad} \mathrm{s}^{-1}$ The system maintains a constant compression at $\approx 1.5 \mathrm{kPa}$ while recording height data at $0.1 \mathrm{~Hz}$. Note that the entire figure consists of a single experiment where all runs are renormalized by their individual values of $\Delta h_{\max }$.

$v$ is grain volume $\left(\sim 10^{-11} \mathrm{~m}^{3}\right.$ for $300 \mu \mathrm{m}$ particles). Thus far, the manipulation of equation (1) has been strictly algebraic and the original definition of the constants was entirely empiric. The recasting, however, suggests thermodynamic interpretations of the parameters. The variable $N$ is the number of grains and $\zeta$ is the average dissipation per grain. Later we will verify these assumptions, in particular the use of $\Theta$, by predicting the configurational entropy of various molecular glass-formers.

The free energy of sand makes two critical predictions as confirmed by experiment. First, microscopically, the constant $\kappa_{1}=v / \Theta$ is an elastic property of the material normalized by the only energy scale ${ }^{8}$ of the system, $\Theta$. Macroscopically, $\kappa_{1}$ is deduced from the experiment ${ }^{2}$ as $\kappa_{1}=-1 / \varepsilon(\mathrm{d} \varepsilon / \mathrm{d} P)_{\dot{\gamma}}$, in a quantity defined as the mechanical compressibility of granular flows. Second, the energy of the flow supplied from the shearing surface is fully dissipated at steady state. The normalized energy, $\kappa_{2} \dot{\gamma}$, would therefore scale as the viscous loss of the flow, $\zeta=v \eta \dot{\gamma}$ where $\eta$ is the effective viscosity of the granular mixture. Comparing the flow of sand and other fluids drained through a funnel $\left(0.25^{\prime \prime}\right.$ opening), we measured a granular viscosity of $\sim 10^{-1} \mathrm{~Pa}$ that matches mineral oil viscosity at room temperature. Using the value ${ }^{8}$ of $\Theta \sim 10^{-7} \mathrm{~J}$, we compute $\kappa_{1} \sim 10^{-4} \mathrm{~Pa}^{-1}$ and $\kappa_{2} \sim 10^{-5} \mathrm{~s}$. These values not only fit to the data of Fig. 1, they also have consistent thermodynamic interpretations.

A unifying theory of jamming must also account for the slow dynamics of glassy liquids. From the volume relaxation of Fig. 2, we observed that sand compacts with a Kolrauch exponent of $\beta \approx 0.6$. Interestingly, typical values of $0.2<\beta<1$ are also observed in molecular glasses near jamming. To unify their dynamics, we recall 


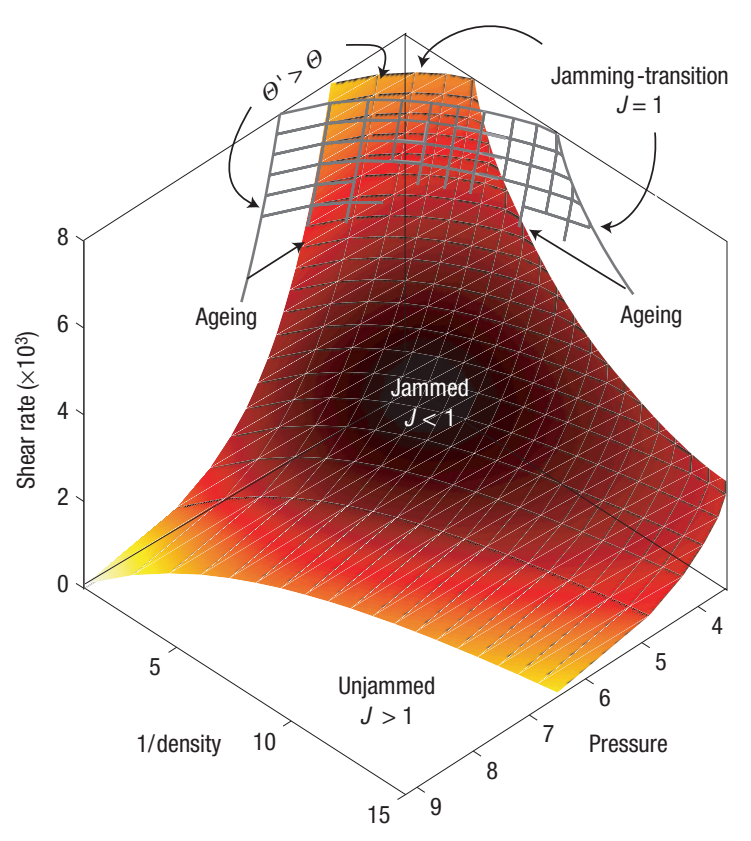

Figure 3 A plot of the concave jamming-transition surface. The shaded surface corresponds to equation (1) by substituting in $\kappa_{1} \equiv v / \Theta$ and $\kappa_{2} \equiv \eta \nu / \Theta$. The jamming parameter, $J(P, \varepsilon, \dot{\gamma}, \Theta)$, is defined by dividing equation (1) by $P(\varepsilon, \dot{\gamma})$. The axes are pressure, $P v / \Theta$, shear rate, $\zeta / \Theta$, and the free volume, $\varepsilon / \varepsilon_{0}$, in normalized forms. The jamming transitions given at $J(\Theta)=1$ (solid) and $J\left(\Theta^{\prime}\right)=1$ (mesh) represent two examples of equilibrium metastable packing arrangements, where the packing at $\Theta$ is denser than the one at $\Theta^{\prime}$ such that $\Theta^{\prime}>\Theta$. The unjammed path initiates above the jamming transition where $J>1$, but terminates at $J=1$ when compaction or structural ageing stops within experimental time. This ensures that the ageing temperature $\Theta$ remains constant so that the values of $\varepsilon_{0}$, $\kappa_{1}$ and $\kappa_{2}$ are stationary in equation (1). The surface is convex if a logarithmic scale is used (see the concavity of the quasi-static fit in Fig. 1).

that a glass is a liquid in which crystallization is bypassed during cooling ${ }^{16}$. This is the exact scenario exhibited by sand; the angular particles jam because the bulk crystallization never nucleates on densification. In light of these similarities, we propose that the EOS of equation (1), as a function of the ageing temperature, encompasses the path-dependent states of both jamming and glass transition. In Fig. 3, two examples of jammed states are shown by two metastable ${ }^{1}$ isothermal surfaces, each defined by a particular ageing temperature, $\Theta$.

To substantiate the above claims, we use Edwards's proposition ${ }^{3}$ that the granular temperature reflects the 'fluffiness' of densely packed grains. To see how 'fluffiness' relates to particle configuration, we derive the entropy difference, $\Delta S_{\text {sand }}$, between the jammed and crystalline states of granular packing. We compute the total entropy $S=-k_{\mathrm{B}}(\mathrm{d} F / \mathrm{d} \Theta)$ from equation (3) and cancel the contributions of the dissipation term in the entropy difference ${ }^{21}$. Conceptually, dissipation is irrelevant to the architectural arrangement of particles. The result is

$$
\Delta S_{\text {sand }}=N k_{\mathrm{B}}-N k_{\mathrm{B}} \ln \left(\varepsilon / \varepsilon_{0}\right) \text {. }
$$

Parameters $P, \varepsilon$ and $\Theta$ are all measured above an ideal jamming condition very near the hypothetical crystalline phase. Therefore, ideally, at the minimum free volume $\varepsilon=\varepsilon_{0}$, $\Delta S_{\text {sand }}=N k_{\mathrm{B}}$ is the communal entropy ${ }^{22}$. (The communal entropy, $k_{\mathrm{B}} N \approx k\left[\ln \left(V^{N} / N !\right)-\ln (V / N)^{N}\right]$ using the Stirling

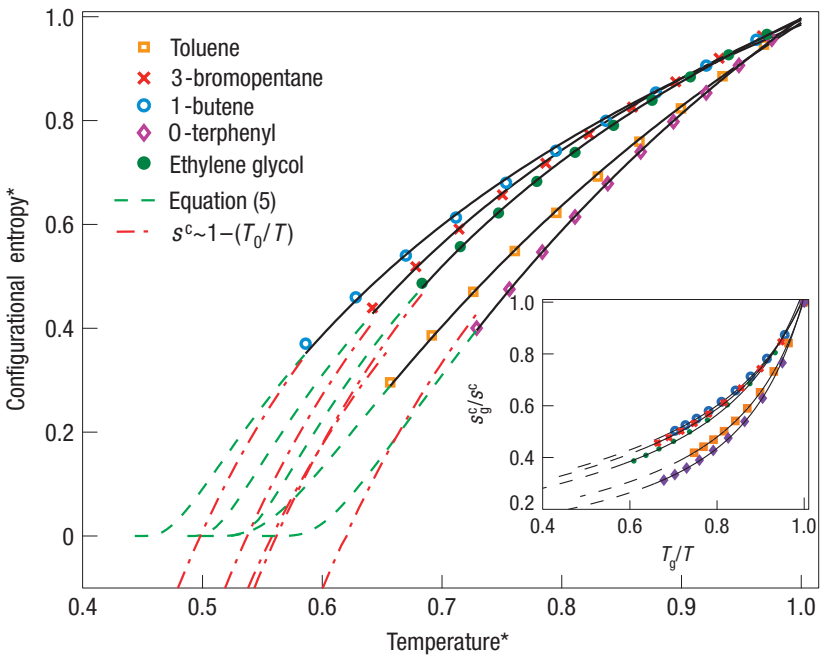

Figure 4 The Kauzmann plot ${ }^{4}$ for five glass-formers of molar configurational entropy ${ }^{*}, s^{c} / S_{\text {fusion }}^{c}$, versus temperature ${ }^{*}, T / T_{\text {fusion }}$, with both quantities normalized by their respective values at the fusion point. The fit agrees well with the entropic data, providing strong evidence for the unification of granular jamming and glass transition. Notably, the entropy averts the Kauzmann paradox (green dashed line), in a prediction unlike the one made by the most elegant glass mode ${ }^{4}$ so far (red dotted-dashed line). Inset: Fragility plot of $S_{\text {glass }}^{\mathrm{c}} / \mathrm{S}^{\mathrm{c}}$ versus $T_{\text {glass }} / T$ for the same five glass-formers, and the dashed line here is not physical being above the fusion point. The fitting function uses equation (5) where the molar entropy, $S^{c}$, is given as $S^{\mathrm{C}}(T) \cong x R \ln \left[1-\exp \left(-\zeta / k\left(T-T_{0}\right)\right)\right]^{-1}$ for $T \geq T_{0}$ and $R$ is the universal gas constant. The values of fragility ${ }^{4,11} x, \zeta / k_{\mathrm{B}}$ and the Kauzmann temperature $T_{0}$, are respectively listed from strong to fragile: $3.24,8.97$ and $45 \mathrm{~K}$ (59.5) for 1-butene ${ }^{26} ; 3.55,17.7$ and $80.6 \mathrm{~K}$ (84.0) for 3-bromopentane ${ }^{27} ; 3.24,30.7$ and $114 \mathrm{~K}$ (153) for ethylene glycol ${ }^{28} ; 4.22,38.9$ and $87.8 \mathrm{~K}(100)$ for toluene ${ }^{29}$; and $6.01,65.5$ and $182 \mathrm{~K}$ (204) for ortho-terpheny ${ }^{30}$. The values in parentheses are the glass-transition temperatures. The matching $x$ between 1-butane and ethylene glycol indicates that the fragility index alone cannot quantify the glass transition completely. The molar configurational entropy is derived using the equation, $S^{\complement}(T)=\Delta_{\text {tusion }} S-\int_{T}^{T_{\text {Tusion }}} \mathrm{d} T^{\prime}\left[\left(C_{\mathrm{p}}^{\text {liq }}-C_{\mathrm{p}}^{\text {cr }}\right) / T^{\prime}\right]$ for $T \leq T_{\text {fusion }}$, and $C_{\mathrm{p}}^{\text {liq }}$ and $C_{\mathrm{p}}^{\text {cr }}$ are the experimentally measured isobaric (molar) heat capacities of the liquid and crystalline states. The interpolation/extrapolation of the heat-capacity data were in terms of second-order polynomials.

approximation $^{21}$, accounts for the entropy difference between a liquid and a solid.) In the case for a non-ideal packing $\left(\varepsilon>\varepsilon_{0}\right)$, however, work must be done to constrain the otherwise purely random particles/molecules to sample only the jammed/glassy states $^{4,18}$ - the possible configurational states for all particles. This work reduces the communal entropy by an amount of the configurational entropy, $S^{c}=N k_{\mathrm{B}} \ln \left(\varepsilon / \varepsilon_{0}\right)$, scaling in proportion to the volume above ideal packing, $\varepsilon$. In other words, as interpreted from equation (4), equally jammed (or fluffy) configurations can be realized for high packing densities as for low ones at the expense of structural order ${ }^{23}$.

To verify the configurational entropy $S^{\mathrm{c}}$, we solve equation (1) for $\ln \left(\varepsilon / \varepsilon_{0}\right)$ so that

$$
S^{c} \cong N k_{\mathrm{B}} \ln [1-\exp (-\zeta / \Theta)]^{-1}
$$

for $C \approx 1$ and $P \ll \Theta / \nu$-which is true for most glasses under atmospheric pressure and thus pressure effects are typically negligible. Figure 4 shows the fit of equation (5) to the configurational entropy data of five different glass-formers. 
At thermal equilibrium, the ageing temperature of equation (5) is rescaled as $\Theta=k_{\mathrm{B}}\left(T-T_{0}\right)$, in terms of the Kauzmann temperature $T_{0}$, to preserve the third law of thermodynamics. The results show a good agreement between theory and experiment in both Kauzmann and fragility plots ${ }^{4}$. Notably, the Kauzmann paradox ${ }^{4,11}$ is entirely averted.

The shear flow experiment of sand has guided a new classification of jamming as a solid-liquid transition uniquely defined at different structural temperatures. The path-dependent transition is purely kinetic, and yet the transition itself is in structural equilibrium with the ageing temperature for $\Theta \geq k_{\mathrm{B}}\left(T-T_{0}\right)$ (ref. 8). In contrast, other variations ${ }^{14,15}$ of the theory rely on an effective granular temperature that is unrelated to the architectural arrangement of particles. Ultimately, the state variables that govern the isothermal states of jamming are pressure, shear rate ${ }^{5}$ and the free volume ${ }^{16}$.

Moreover, the EOS for dense granular flows has provided strong evidence for the unification of jamming in fragile materials. Broadly speaking, it considers the elastic, the entropic, the free volume and the hydrodynamic bases of other glass theories presented so far. This view of jamming applies to phenomena such as stick-slip nucleation in seismic fault ruptures ${ }^{24}$, shear banding in metallic alloys $^{5}$, strain softening in colloidal glasses ${ }^{6}$ and even stop-and-go driving in traffic jams $\mathrm{s}^{25}$. These types of flow, defiant of conservative fluid models, are closely governed by dynamics that straddle the tipping point of jamming.

Received 5 September 2007; accepted 5 March 2008; published 6 April 2008.

\section{References}

1. Cates, M. E., Wittmer, J. P., Bouchaud, J. P. \& Claudin, P. Jamming, force chains, and fragile matterPhys. Rev. Lett. 81, 1841-1844 (1998).

2. Lu, K., Brodsky, E. E. \& Kavehpour, H. P. Shear-weakening of the transitional regime for granular flow. J. Fluid Mech. 587, 347-372 (2007).

3. Edwards, S. F. \& Oakeshott, R. B. S. Theory of powders. Physica A 157, 1080-1090 (1989).

4. Debenedetti, P. G. \& Stillinger, F. H. Supercooled liquids and the glass transition. Nature 410, 259-267 (2001).

5. Demetriou, M. D. et al. Cooperative shear model for the rheology of glass-forming metallic liquids. Phys. Rev. Lett. 97, 065502 (2006)

6. Kobelev, V. \& Schweizer, K. S. Strain softening, yielding, and shear thinning in glassy colloidal suspensions. Phys. Rev. E 71, 021401 (2005).
7. Lewandowski, J. J. \& Greer, A. L. Temperature rise at shear bands in metallic glasses. Nature Mater. 5, 15-18 (2006).

8. Wang, P., Song, C. M. \& Makse, H. A. Dynamic particle tracking reveals the ageing temperature of a colloidal glass. Nature Phys. 2, 526-531 (2006).

9. Lohse, D. et al. Impact on soft sand: Void collapse and jet formation. Phys. Rev. Lett. 93, 198003 (2004).

10. Nowak, E. R., Knight, J. B., Povinelli, M. L., Jaeger, H. M. \& Nagel, S. R. Reversibility and irreversibility in the packing of vibrated granular material. Powder Technol. 94, 79-83 (1997).

11. Angell, C. A. Formation of glasses from liquids and biopolymers. Science 267, 1924-1935 (1995).

12. D'Anna, G. \& Gremaud, G. The jamming route to the glass state in weakly perturbed granular media. Nature 413, 407-409 (2001).

13. Ono, I. K. et al. Effective temperatures of a driven system near jamming. Phys. Rev. Lett. 89, 095703 (2002).

14. Trappe, V., Prasad, V., Cipelletti, L., Segre, P. N. \& Weitz, D. A. Jamming phase diagram for attractive particles. Nature 411, 772-775 (2001)

15. Langer, S. A. \& Liu, A. J. Sheared foam as a supercooled liquid? Europhys. Lett. 49, 68-74 (2000).

16. Turnbull, D. \& Cohen, M. H. Free-volume model of amorphous phase - glass transition. J. Chem Phys. 34, 120 (1961).

17. Savage, S. B. Analyses of slow high-concentration flows of granular materials. J. Fluid Mech. 377, 1-26 (1998).

18. Sastry, S., Debenedetti, P. G. \& Stillinger, F. H. Signatures of distinct dynamical regimes in the energy landscape of a glass-forming liquid. Nature 393, 554-557 (1998).

19. Onoda, G. Y. \& Liniger, E. G. Random loose packings of uniform spheres and the dilatancy onset. Phys. Rev. Lett. 64, 2727-2730 (1990)

20. Song, C. M., Wang, P. \& Makse, H. A. Experimental measurement of an effective temperature for jammed granular materials. Proc. Natl Acad. Sci. USA 102, 2299-2304 (2005).

21. Reif, F. Fundamentals of Statistical and Thermal Physics (McGraw-Hill, New York, 1965).

22. Hirschfelder, J., Stevenson, D. \& Eyring, H. A theory of liquid structure. J. Chem. Phys. 5, 896-912 (1937)

23. Wei, J. et al. Molecular Modeling and Theory in Chemical Engineering Vol. 28 (Academic, New York, 2001)

24. Scholz, C. H. Earthquakes and friction laws. Nature 391, 37-42 (1998).

25. Nagatani, T. Gas kinetics of traffic jam. J. Phys. Soc. Japan 66, 1219-1224 (1997).

26. Takeda, K., Yamamuro, O. \& Suga, H. Thermodynamic study of 1-butene-exothermic and endothermic enthalpy relaxations near the glass-transition. J. Phys. Chem. Solids 52, 607-615 (1991).

27. Takahara, S., Yamamuro, O. \& Matsuo, T. Calorimetric study of 3-bromopentane-correlation between structural relaxation-time and configurational entropy. J. Phys. Chem. 99, 9589-9592 (1995).

28. Takeda, K., Yamamuro, O., Tsukushi, I., Matsuo, T. \& Suga, H. Calorimetric study of ethylene glycol and 1,3-propanediol: Configurational entropy in supercooled polyalcohols. J. Mol. Struct. 479 227-235 (1999)

29. Yamamuro, O. et al. Calorimetric study of glassy and liquid toluene and ethylbenzene: Thermodynamic approach to spatial heterogeneity in glass-forming molecular liquids. J. Phys. Chem. B 102, 1605-1609 (1998).

30. Chang, S. S. \& Bestul, A. B. Heat-capacity and thermodynamic properties of ortho-terphenyl crystal, glass, and liquid. J. Chem. Phys. 56, 503 (1972).

\section{Acknowledgements}

This work is partially supported by the Airforce Office of Scientific Research (Surface \& Interfacial Science program, Grant number FA9550-07-1-0324)

Author information

Reprints and permission information is available online at http://npg.nature.com/reprintsandpermissions. Correspondence and requests for materials should be addressed to H.P.K. 